\title{
CLIMA ÉTICO CORPORATIVO: Una aplicación del modelo de Victor y Cullen en las empresas del estado de São Paulo*
}

\author{
Elio Ferrato \\ UNIMEP-PIRACICABA (BRASIL) \\ kandra@uol.com.br \\ Maria Cecilia Coutinho de Arruda \\ FGV-EAESP (BRASIL) \\ carruda@fgvsp.br
}

\section{Resumen}

En el año de 1988, Victor y Cullen desarrollaron una investigación empírica para medir el clima ético de trabajo de 872 empleados de cuatro empresas norteamericanas. Basados en algunos de los parámetros señalados por dichos autores, el presente estudio tuvo el propósito de analizar el clima ético en nueve corporaciones brasileñas, ubicadas en el estado de São Paulo, Brasil, abarcando a 2573 funcionarios. La investigación brasileña sugiere la existencia de diferentes tipos de climas éticos en las corporaciones y una diferenciación en función de las normas difundidas por el cuerpo directivo de dichas organizaciones. No fue el objeto de esta investigación (la brasileña) establecer una teoría sobre el clima ético, como lo hicieran Victor y Cullen; pero se discutieron las implicancias de las teorías sobre clima ético en las organizaciones.

Palabras clave: clima ético corporativo, alcances del clima ético, teoría de las organizaciones.

\begin{abstract}
Victor and Cullen (1988) developed an empirical survey in order to measure the ethical climate in the organizational bases of 872 employees of four North-American companies. Based on some of the parameters pointed out by these authors, this study had the objective of analyzing ethical corporate climates in nine Brazilian organizations, located in the São Paulo State, Brazil, involving 2573 employees. The Brazilian survey suggested the existence of distinct types of ethical climate and a differentiation in function of norms spread out by the board of directors in the organizations. It was not the purpose to establish a theory about ethical climate, as done by Victor and Cullen, nevertheless, implications of ethical corporate climate in organization theories have been discussed.
\end{abstract}

Key words: ethical corporate climate, ethical climate dimensions, organizations theory.

* Una versión anterior de este trabajo fue publicada en los Anales del VIII Congreso de la Asociación Latinoamericana de Ética, Negocios y Economía (Alene), llevado a cabo el 11 y 12 de agosto de 2005 en Valparaíso, Chile. Traducido del portugués para esta edición por Rosario Madueño Díaz. 


\section{Introducción}

De acuerdo con la investigación de Victor y Cullen (1988: 101), cada vez es mayor la concepción de que las organizaciones son los actores sociales responsables por el comportamiento ético y antiético de sus empleados. Actualmente, esta posición debe ser válida para el mercado norteamericano. Sin embargo, el mercado brasileño, en especial el estado de São Paulo, objeto del presente estudio, amerita otros análisis que trasciendan a las organizaciones vistas solamente como actores sociales responsables por el comportamiento ético y antiético de sus empleados, como se apreciará a lo largo de esta investigación.

Victor y Cullen afirman, incluso, que dicha perspectiva se refleja en los juicios legales contra las corporaciones, especialmente cuando se trata de delitos corporativos, de manera que académicos y profesionales han mostrado creciente interés en la comprensión y el gerenciamiento de los sistemas normativos de las organizaciones que puedan guiar $\mathrm{u}$ orientar el comportamiento ético de los empleados. Existen pocas herramientas teóricas y metodológicas capaces de establecer normas a nivel organizacional.Un mecanismo conveniente para entender los sistemas normativos de las organizaciones es el concepto de clima en el trabajo (Schneider, 1983, citado por Victor y Cullen), que en este documento se denomina clima ético en las organizaciones.

El clima ético es definido por Schneider (1975: 474) como percepciones, que son «descripciones importantes de significados psicológicos que las personas pueden concordar en caracterizar como procedimientos y prácticas de un sistema». Las percepciones predominantes sobre las prácticas y procedimientos organizacionales típicos constituyen el clima ético en el trabajo, de ahí que el interés de esta investigación sea conocer cómo ocurre este proceso.

Los casos relativamente recientes de Enron en Estados Unidos, de Baring Bank en Singapur, entre otros, motivaron nuevos estudios al respecto; se trataba de identificar a los principales actores sociales responsables de las actitudes antiéticas de los funcionarios y ejecutivos de las organizaciones. Además de la catástrofe financiera, esas actitudes antiéticas mancharon la imagen y la marca de las corporaciones $\mathrm{y}$, en consecuencia, pueden echar por tierra, y para toda la vida, la reputación de sus representantes legales.

Otros autores, desde otros enfoques, trataron el clima ético corporativo. Cabe citar a De George (1995), que representó el clima ético por los valores, las prácticas y procedimientos que involucran comportamientos y actitudes morales de la organización. Al analizar la ética empresarial, De George (1995) estudió el contenido moral de las decisiones, el proceso y la acción práctica de tales decisiones y cómo eso debe ocurrir en la dinámica de las relaciones entre los individuos dentro de las organizaciones y de estas con los stakeholders. A semejanza de lo que ocurre con la ética empresarial, el clima ético proporciona el contenido moral de las decisiones (es decir, lo que debe ser hecho), el proceso y la práctica de esas decisiones (cómo deben llevarse a cabo) en las relaciones entre las personas dentro de la institución y en la interacción de esta con sus stakeholders.

Arruda y Navran (2000) desarrollaron indicadores del clima ético, adaptando 
para el Brasil el modelo de Frank Navran, en asociación con el Ethics Resource Center. Aguilar (1996) define la empresa ética como aquella que ha conquistado el respeto y la confianza de sus stakeholders, estableciendo un equilibrio aceptable entre sus intereses económicos y los intereses de todas las partes directa o indirectamente afectadas por la toma de decisiones.

El objetivo de este trabajo fue alcanzar una mejor comprensión del clima ético corporativo en las empresas brasileñas. Para este fin, se tomó el estado de São Paulo como centro del estudio. A partir de la investigación, se trató de establecer si el clima ético observado difiere considerablemente del descrito por Victor y Cullen. El estudio de Victor y Cullen describe a la sociedad norteamericana como dotada de caracteres muy diferentes de los de la sociedad brasileña, en su historia, tradición e ideas de nuevos proyectos.

\section{Climas de trabajo y organizaciones éticas}

De acuerdo con Schneider (1975), no hay un clima de trabajo común a todas las empresas. Los investigadores han estudiado con mucha frecuencia la existencia y la amplitud del control y de la autonomía, el grado de la estructura, la naturaleza de las recompensas, el estímulo y el soporte en las organizaciones; y la mayoría de estos tipos de clima corresponden a dos grandes clasificaciones:

a) Opiniones generales sobre las normas organizacionales relacionadas con las formas de estructura y procedimientos para el empleo de recompensas y controles. b) Opiniones generales respecto de normas organizacionales que proporcionen valores de soporte, tales como: entusiasmo, apoyo a los colegas y a los subordinados.

Algunos de los tipos de clima representados por normas organizacionales tienen una base ética, mediante las cuales se informa a los miembros de la organización lo que las personas pueden hacer o lo que deben hacer con relación al trato con otras personas.

Los tipos de climas que brindan ayuda para la resolución de conflictos y para la no aceptación de la agresión representan normas de base ética, percibidas sea por organizaciones o por grupos. Sin embargo, en la literatura sobre organizaciones, las investigaciones anteriores no se basaron explícitamente en una base teórica o filosófica para seleccionar los tipos de normas o estudios éticos.

Victor y Cullen (1988) se basan en la filosofía ética y en las teorías del comportamiento ético -siendo estas últimas las que sensibilizan a la sociedad norteamericana- para la selección de los temas que constituyen la base de los climas éticos en el trabajo. Sus premisas difieren mucho de las de este estudio, en la medida en que factores como la cultura organizacional brasileña, aun incluyendo en ella a las multinacionales europeas, representa un escenario particular y general diferenciado del perfil norteamericano.

La condición básica para que haya un clima ético en las organizaciones es que los sistemas normativos formales estén establecidos; es decir, que los empleados o los funcionarios deben percibir los estándares normativos de la organización 
con un grado aceptable de consenso. Así, en la metodología utilizada en la investigación, se solicita a los empleados de las organizaciones que no se refieran a sus propios valores y conductas, sino que tomen en cuenta las prácticas y procedimientos que ellos perciben que existen en sus organizaciones. De esta forma, los entrevistados están vistos aquí como un tipo de observador que difiere mucho de aquellos observadores antropológicos que son miembros de la cultura organizacional y proporcionan la visión desde dentro de la empresa, como sugieren Victor y Cullen.

Un sistema normativo institucionalizado en la organización puede ser eventualmente considerado un elemento de la cultura; a pesar de que la cultura organizacional es ciertamente más amplia, al incluir, por ejemplo, estándares de comportamientos, de actitudes y de comunicación, entre otros. Los estudiosos de climas éticos en las organizaciones se ocupan solamente de lo relacionado con las normas empresariales y lo que estas expresen respecto a prácticas y procedimientos de consecuencias éticas; por tanto, abordan apenas una faceta de la cultura de las organizaciones.

El cuestionario para el estudio del clima ético en el trabajo es, entonces, apenas un instrumento a través del cual se manifiestan las percepciones de los empleados y las dimensiones éticas que retratan la cultura de una organización.

Deal y Kennedy (1982, citados por Victor y Cullen) afirman que las culturas organizacionales «más desarrolladas» producen un mayor consenso en las percepciones de los climas (éticos) de trabajo.

\section{Tipos de climas éticos corporativos}

Para el presente estudio se emplearon dos tipologías de climas corporativos éticos, tomando como modelo la línea aplicada por Victor y Cullen. La primera tipología del clima ético se refiere al criterio ético, que indica los principios de comportamiento para la toma de decisiones en la organización, es decir, el egoísmo, la benevolencia y el principio, que puede ser interpretado aquí como carácter. La segunda tipología del clima ético trata del centro de análisis, es decir, dónde se toman las decisiones: el individuo, que se manifiesta en lo personal; el conjunto de individuos de la organización, expresado en lo local; o la organización en el contexto social, que se refleja en lo universal.

El cruce o interrelación de estas dos tipologías de clima ético abre un espectro de nueve tipos teóricos de climas éticos corporativos, como los que se muestran en la figura 1 (ver página siguiente).

\subsection{Centro del análisis y criterio ético}

Las dos coordenadas de la figura 1, que hacen referencia a las tipologías de los climas éticos, el centro de análisis y el criterio ético, se articulan en nueve cuadrantes. El centro de análisis, ampliando lo ya expuesto anteriormente, trata de un grupo de referencias para identificar la fuente del pensamiento moral empleado para aplicar los criterios éticos (Fritzche y Becker, 1984) en las decisiones organizacionales y/o los límites sobre lo que debería ser considerado en el análisis ético de las decisiones organizacionales.

El primer cuadrante que resulta de cruzar el centro de análisis con el criterio 
CENTRO DE ANÁLISIS

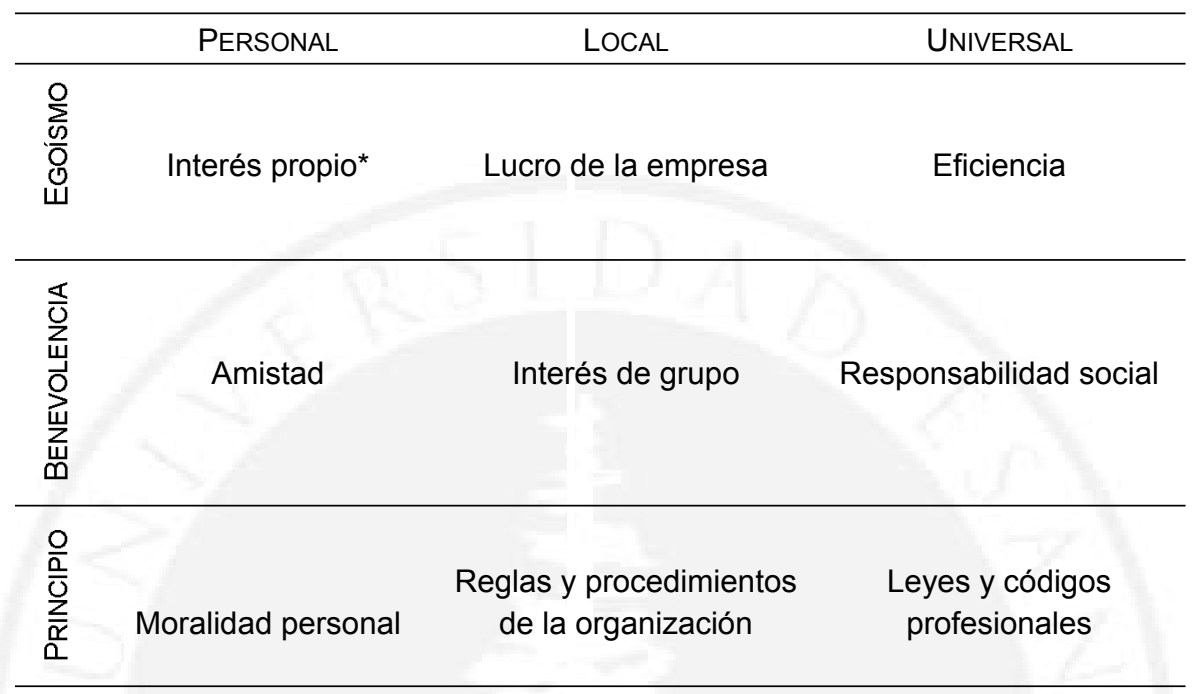

Figura 1. Tipos teóricos de climas corporativos éticos

ético es el interés propio. Este indica el elemento particular del esfuerzo personal y egoísta, que induce y dirige todo a la propia satisfacción, en detrimento del resto del grupo y de la compañía.

El cuadrante de la amistad, derivado de la variable personal y la benevolencia, sugiere el desprendimiento personal del egoísmo para atender a un llamado humanitario, con un espíritu solidario de lucha por el interés del grupo.

El cuadrante de la moralidad personal, derivado de las variables personal y principio, tiene como objetivo el hacer o conducirse de acuerdo con el propio carácter, dotado de principios o del principio de honradez.

El cuadrante del lucro o beneficio de la empresa, derivado de las variables local y egoísmo, enfoca el esfuerzo valorado por la empresa. Quienes participan en este cuadrante luchan por la maximización de los lucros y beneficios en pro de la organización.

El cuadrante del interés de grupo, derivado de las variables local y benevolencia, orienta las acciones y expresiones en pro del grupo, al cual el individuo piensa pertenecer.

El cuadrante de reglas y procedimientos de la organización, derivado de las variables local y principio, indica que las actitudes son tamizadas por las normas y reglas establecidas por la empresa y son orientadas por principios de ética a partir del grupo.

El cuadrante de eficiencia, derivado de las variables universal y egoísmo, denota una visión del ambiente macrosocial donde la organización se inserta, de modo que a través de la eficiencia la organización conquista su lugar en el mercado, sin la intención de compartir los logros adquiridos con otras empresas. 
El cuadrante de la responsabilidad social, derivado de las variables universal y benevolencia, tiene por fin establecer esfuerzos para que la comunidad se beneficie del clima preponderante en aquella organización.

El último cuadrante, de las leyes y códigos profesionales, derivado de las variables universal y principio, abarca toda acción adoptada por los empleados, las que deben alinearse con las reglas y los códigos profesionales; donde el carácter general sigue una línea específica de principios morales, totalmente desinteresada de las cuestiones del ámbito personal o grupal, en beneficio de la sociedad donde se inserta la firma.

Para reunir la información relativa a las dos tipologías de climas éticos en las compañías, fue necesario el estudio de cinco dimensiones del clima ético, cuyo análisis puede ser visto en la investigación de campo presentada más adelante: interés (caring), ley y código profesionales (law and code), reglas (rules), instrumentos (medios) y autonomía. Los aspectos que requieren más aclaraciones aquí son: los instrumentos (medios) y la autonomía.

El aspecto instrumental (medios) se refiere a la forma de obtención de las ganancias personales y también al lucro de la organización. El aspecto de la autonomía se relaciona con la propia ética adoptada por el individuo, el grupo o la empresa en el contexto social.

\subsection{Algunos pensamientos complementarios sobre los climas éticos}

Basados en el modelo teórico presentado es razonable pensar que el clima ético es un concepto organizacional o de grupo; no se asume, por lo tanto, la secuencia evolutiva de la individualidad. Estudios anteriores comprobaron que el argumento ético individual es incompatible con el concepto de clima ético organizacional. La combinación entre las variables «X»e «y» (véase figura 1) produce diferentes resultados en función de los factores culturales, económicos y políticos de la comunidad donde se inserta el grupo de empresas estudiadas; de ahí que la razón de este trabajo sea proponer un aprendizaje teórico, para lo cual pueden formularse diversas teorías o conceptos, como los descritos.

Algunas de las hipótesis que pueden formularse a partir de las combinaciones (de los cuadrantes) indicadas anteriormente son las siguientes: Las personas benevolentes tienden a ser menos receptivas o predispuestas a conducirse de acuerdo con leyes y reglas, y también pueden ser menos abiertas a argumentos basados en reglas o principios. En contraste, las personas que están formadas en principios, de carácter, tienden a ser menos sensibles a los efectos particulares u específicos que puedan causarse en otras personas.

En función de estos datos, se puede también esperar que las organizaciones desarrollen, en términos relativos, distintos climas éticos. Ello significa que organizaciones o subgrupos pueden ser caracterizados como prototipos de benevolentes, basados en los criterios éticos de egoísmo o de carácter. A pesar de que Victor y Cullen han detectado en sus investigaciones que siempre hay un tipo de clima dominante en una organización o grupo, ninguna organización tiene un tipo de clima exactamente igual al del otro. 
Los tipos de climas éticos existentes en una organización o grupo influyen en la resolución de lo que se considera conflicto ético. Estos conflictos pueden ser asociados con el clima moral y social de una organización, cuyo impacto es significativo en la toma de decisiones de las personas. En un clima ético de principios, la aplicación e interpretación de las reglas y leyes pueden ser la forma dominante de pensamiento. Por otro lado, en un clima típicamente egoísta, el interés propio debe ganar posiciones dominantes.

A diferencia de la filosofía y el desarrollo moral del individuo, el clima ético refleja el concepto de la organización; por lo tanto, el pensamiento ético puede variar en función del concepto de una organización.

El centro del análisis fue obtenido de teorías de trabajos sociológicos y de grupos de referencias. Merton y otros, al estudiar los actores de los sistemas sociales, identificaron tipos de grupos de referencia que ayudan a moldear los comportamientos y actitudes de los actores. Merton (1957) consiguió establecer la distinción entre el papel local y el papel universal. Para los actores locales, los grupos de referencia importantes o fuentes de definición de roles y expectativas están comprendidos en un sistema social. Para los actores universales, las fuentes de definición de sus roles residen en un sistema social externo al sistema al cual ellos pertenecen.

En la tipología del clima ético, las fuentes de pensamiento ético para el centro del análisis están dentro de la organización, como el grupo de trabajo. En el nivel universal, las fuentes de pensamiento ético son externas a la organización analizada, tales como una asociación profesional cualquiera. Las fuentes «universales» del pensamiento ético pueden ser conceptos abstractos generados fuera de las organizaciones, pero utilizados dentro de las mismas como parte de un sistema normativo institucionalizado.

Las fuentes de las normas profesionales de comportamiento, por ejemplo, se encuentran usualmente fuera del ambiente de trabajo. Mientras que estas normas todavía son parte del clima normativo prevaleciente en las organizaciones de trabajo profesional.

A pesar de que los centros (loci) de análisis identifican las fuentes de pensamiento y los límites de las reflexiones en los análisis éticos, las relaciones entre los centros de análisis y los criterios éticos difieren considerablemente para cada elemento involucrado en estas relaciones según su idiosincrasia.

Como se observa en la figura 1 , en el contexto de la variable egoísta, el centro (centros) de análisis personal identifica lo específico del mismo yo. Por ejemplo, una empresa individual que supuestamente va a actuar a favor de alguien en particular. En el locus (centro) del análisis personal, el criterio egoísta es definido como la consideración de las necesidades y las preferencias de alguien (de él mismo); por ejemplo, la ganancia personal y defensa propia. En el caso del locus (centro) del análisis local, se define el interés de la organización; por ejemplo, el lucro, la ventaja estratégica. Finalmente en el locus (centro) universal, la definición se orienta a la consideración de los intereses ampliados de los sistemas sociales y económicos; por ejemplo, la eficiencia. 
En el contexto de la variable benevolencia, el locus (centro) del análisis identifica a los miembros de la organización, preguntandoquiénes somos y establece los límites para nuestras responsabilidades. Esta distinción del sujeto-objeto es concomitantemente obligatoria para con los otros, diferenciando al benevolente del egoísta. En el centro de análisis personal, el criterio de benevolencia se define como la consideración con otras personas; sin hacer referencia a la participación en la organización: por ejemplo, la amistad y la reciprocidad.

En el centro (locus) de análisis local, se considera a la organización de manera colectiva, por ejemplo, el espíritu de equipo, jugar en el equipo.

En el centro de análisis universal (locus), la benevolencia se define como la consideración hacia los otros reflejada en otras formas de acción externas a la organización; por ejemplo, la responsabilidad social.

En el contexto de la variable principio, el centro de análisis define las fuentes de los principios que se esperan sean usados en la organización.

En el centro de análisis personal, los principios son escogidos por sí mismos, es decir, se espera que las personas sean guiadas por la ética personal.

En el centro del análisis local, la fuente de principios permanece dentro de la organización; por ejemplo, reglas y procedimientos.

En el centro de análisis universal, la fuente de los principios es extraorganizacional, está fuera de la organización; por ejemplo, el sistema legal, las organizaciones profesionales. En los climas local y universal, las personas son guiadas por fuentes de principios independientemente del individuo; por lo tanto, no hacen referencia a las preferencias éticas personales de cada uno.

\subsection{Las bases organizacionales del clima ético en el trabajo}

Los investigadores del clima corporativo se enfocan en dos temas límites principales, referidos a la construcción del clima ético: uno es el nivel de agregación y el segundo corresponde a la creencia, los cuales afectan los componentes del clima ético. El clima organizacional, según el punto de vista de Reichers y de Schneider (1990), puede ser entendido como la percepción compartida de los empleados con respecto a las políticas, prácticas y procedimientos formales e informales de la organización.

Por lo tanto, el clima grupal u organizacional es la forma agregada típicamente percibida por sus funcionarios o empleados. La forma agregada está constituida por una serie de factores que pueden ser identificados como variables que incluyen personas, situaciones cambiantes y la combinación de persona-situación. La variable personas se refiere a datos demográficos individuales, como edad y sexo, y se refleja en los componentes de la organización; por ejemplo, grupos de trabajo.

Las variables combinadas personasituación se caracterizan como variables personales con relación a factores organizacionales tales como salarios y compensaciones. Ya que el objetivo de esta investigación es saber si los climas en el trabajo tienen bases interorganizacionales y/o 
intraorganizacionales, se analizaron las variables situaciones variables y la combinación persona-situación. De este modo, el principal interés de este trabajo fue determinar si el clima ético se manifiesta entre las diferentes organizaciones y/o al interior de estas, a partir de sectores definidos por las compañías investigadas.

El segundo tema del clima ético se refiere a la creencia, que abarca la formación social y educacional del individuo y del grupo de una organización específica, lo que se manifiesta a través de los contactos interpersonales de la organización con otras compañías y el contexto social en el cual se inserta.

\section{Investigación de campo}

Tanto en el estudio de Victor y Cullen como en el estudio elaborado en el estado de São Paulo se percibió la preocupación de los directivos acerca de la confidencialidad de sus declaraciones. Como una forma de aliviar las tensiones y recelos con relación al hecho de hacer públicos los datos recogidos en las investigaciones, se garantizó una total y absoluta discreción en cuanto a los nombres o indicios que pudieran revelar la identidad de las empresas involucradas; tarea que no fue fácil en un primer momento.

Para este estudio se seleccionaron nueve compañías de las ramas de alimentos, embalajes, metalurgia, logística, autopartes, maderas, filtros industriales y distribución de bienes, cuyo número de funcionarios variaba entre 75 y 1140 personas, ubicadas en las regiones de Indaiatuba, Monte Mor y Jundiaí, pertenecientes al estado de São Paulo. Los gerentes de estas empresas recibieron personalmente un cuestionario explicando el objetivo del estudio. Copias de este cuestionario se entregaron internamente a los jefes de sección, quienes se responsabilizaron por la devolución de los cuestionarios llenados, de manera que el control de las entregas y devoluciones de los formularios llenados ocurrió sin dificultades.

Lo que facilitó la distribución, el llenado y la devolución de los cuestionarios es la relativa frecuencia con que las empresas tienen que entregar información, especialmente con miras a la obtención de la certificación técnica, del tipo ISO 9000, o hasta para obtener el premio nacional de calidad (PNC), hoy bastante difundido entre las empresas que buscan su inserción en el mercado nacional e internacional. A pesar de que el llenado y la devolución de los formularios no era obligatorio y de que se garantizó la confidencialidad de la encuesta, hubo un $23 \%$ de cuestionarios no devueltos. Los encargados de las jefaturas señalaron que el plazo fue muy corto. Aun así, el número de respuestas obtenidas fue considerado un buen resultado. De un total de 2573 funcionarios, el $77 \%$, es decir, 1981 dedicaron su tiempo a responder el cuestionario, que trataba sobre las cinco dimensiones del clima ético: interés (caring), ley y códigos profesionales (law and code), reglas (rules), instrumental (medios) e independencia.

La investigación preveía inicialmente aplicar la investigación por departamentos de cada organización y según sexo, formación educacional y edad de los encuestados, lo que hubiese enriquecido grandemente los resultados. Sin embargo, finalmente esto no se llevó a cabo con el fin de reforzar la confidencialidad de las respuestas, aspecto de gran preocupación para los directivos de las empresas. 


\subsection{Medición del clima corporativo}

El cuestionario del clima ético, basado en el formulación de Victor y Cullen, registra las opiniones de los encuestados en cuanto a cómo los miembros de las respectivas organizaciones toman decisiones relacionadas con hechos, eventos, prácticas y procedimientos que requieren criterios éticos. El detalle de cada ítem sobre el clima ético tuvo el objetivo de abarcar los nueve cuadrantes derivados del centro del análisis y del criterio ético, descritos anteriormente. Cada uno de esos tipos representó a priori una clase de comportamiento ético en la organización.

En esta investigación, el clima ético se refirió más a la forma de exponer la ética y a la estructura de los procesos en la toma de decisiones, que al propio contenido, esto es, a la diversidad de valores en los resultados de la decisión.

A los encuestados se les solicitó indicar en una escala Likert de siete puntos, cuáles de los ítems describía con precisión su clima de trabajo. La escala de siete puntos tuvo varias anclas verbales: completamente falso (0), mayormente falso (1), parcialmente falso o de alguna forma falso (2), ni verdadero ni falso (3), parcialmente verdadero o en alguna forma verdadero (4), mayormente verdadero (5) y completamente verdadero (6).

En el presente caso, los comportamientos, procedimientos y hechos fueron escogidos para reflejar el uso del criterio ético en la toma de decisiones empresariales. Mediante este cuestionario se puede presuponer que, por lo menos en promedio, los encuestados pueden actuar como observadores organizacionales objetivos, a pesar de que sus percepciones son filtradas por características psicológicas individuales y otras diferencias personales y, por tanto, sus respuestas valorativas o afectivas podrían ser confundidas con percepciones del clima ético. Para evitar este problema, el estudio desarrolló preguntas que dieran énfasis a la descripción del hecho en vez de a sentimientos. De este modo, el cuestionario no se enfocó en el hecho de que el encuestado demostrara que él o ella se comportaban éticamente ni si consideraba el clima ético como bueno o malo. (Victor y Cullen, 1987: 58).

\subsection{Resultados}

Los ítems que describen el conjunto de los nueve tipos teóricos del clima ético fueron analizados de acuerdo con causas de rotación, siguiendo el análisis de los factores desarrollado por Abdi (2003), los cuales abarcan datos de las nueve empresas estudiadas, teniendo como punto de partida las cinco dimensiones de los climas éticos. Este estudio es semejante, en cierta forma, al desarrollado por Victor y Cullen (1987); no obstante, para cada empresa investigada en el estado de São Paulo se asumió un valor medio para cada combinación de las variables criterio ético y centro de análisis (locus) versus las cinco dimensiones, representando así las 1981 respuestas de los empleados estudiados.

El análisis de los datos abarca en primera instancia, el estudio del factor exploratorio y el análisis de ítems versus factores de respuestas en la escala de Likert, sin tener en cuenta la escala reducida para obtener un mayor índice de correlación de los ítems en el total. No se consideró el grado de confiabilidad, del tipo a $=0,70$ por ejemplo, para hacer lo suficientemente consistentes las evidencias, y finalmente sin grado de distinción para medir la ela- 
boración de la escala, como se hizo en los estudios del Departamento de Defensa Nacional del Canadá, realizados por Kelloway et ál. (1999), respaldados por los estudios de Victor y Cullen (1988). En un futuro estudio de esta naturaleza se piensa tomar en cuenta las tres variables indicadas anteriormente.

El cuadro 1 (ver página siguiente) presenta las variables y datos para las cinco dimensiones de los climas éticos y los ítems que pretenden describir las combinaciones de los centros de análisis y los criterios éticos expuestos en la figura 1 . Las abreviaciones al final del cuadro 1 identifican esas combinaciones de las dimensiones de los climas éticos, por ejemplo, el ítem con la sigla «BL» se refiere a la intersección entre las variables local y benevolencia de la figura 1 .

Se estableció que hay una relativa tendencia a la concentración en los factores 3, 4 y 5 en los ítems: interés, leyes y códigos profesionales, reglas e independencia. En el ítem de instrumentos (medios) esa concentración quedó diluida en relación con los demás.

Ray (1973: 11) sugiere que el análisis de los factores debe ser realizado con mucha calma, dadas las múltiples interpretaciones que son posibles a partir de las combinaciones de las variables analizadas en una determinada investigación. Para este autor, cuando hay un número elevado de características de un grupo de personas, se tiende a analizar la relación entre esas características en términos de una matriz de intercorrelación.

Ray determina que incluso si hubiese diez características para un grupo de personas, habrá una matriz de 45 coeficien- tes de correlación. El análisis del factor es una tentativa de reducir esta matriz a dimensiones más manejables y de mejor interpretación. Si fuera posible reducir de diez a tres variables básicas, entonces las correlaciones de la interpretación solicitada se reducirían de 45 a sólo 3 . Una alternativa para llegar a ese resultado es el análisis conceptual. Ejemplificando, se puede decir que tres variables, como ocupación, renta y educación, pueden ser reducidas a una variable de clase. Como en esta investigación no fue posible juntar un conjunto de variables, se usó el análisis de factores. Las variables principio, benevolencia y egoísmo, en el eje de la ordenadas de los criterios de la ética, quedarán reducidas a comportamientos. Las variables personal, local y universal, en el eje de las abcisas del centro de análisis, quedarán reducidas a personas. De esta forma, el análisis del factor se redujo de aproximadamente 40 coeficientes de correlación a apenas dos, para cada ítem de la investigación.

El análisis de los resultados de la investigación también tomó en consideración la media aritmética para cada grupo de ítem investigado (interés, leyes y códigos profesionales, reglas, instrumental e independencia), y las respuestas de cada grupo en la escala Likert ${ }^{1}$ de 0 a 7 , distri-

1. Nota de traducción:

El tipo de escala aditiva más frecuentemente utilizado en el estudio de las actitudes sociales es el de Likert. La escala de Likert es ordinal y como tal no mide en cuánto es más favorable o desfavorable una actitud; es decir, si una persona obtiene una puntuación de 60 puntos en una escala, no significa esto que su actitud hacia el fenómeno medido sea el doble que la de otro individuo que obtenga 30 puntos, pero sí nos informa que el que obtiene 60 puntos tiene una actitud más favorable que el que tiene 30 , de la misma forma que $40{ }^{\circ} \mathrm{C}$ no son el doble de $20^{\circ} \mathrm{C}$ pero sí indican una temperatura más alta. 


\section{Cuadro 1: Factores de rotación e ítems}

\begin{tabular}{lllllll}
\hline Ítems & Factores & & & \\
\hline & 1 & 2 & 3 & 4 & 5 \\
\hline
\end{tabular}

1. Interés (caring)

(BL) El factor más considerado aquí es el mejor para todos en la compañía.

$\begin{array}{lllll}1 & 0 & 12 & 8 & 10\end{array}$

(BL) La mayor preocupación es el bienestar de todas las personas de la empresa como un todo $\quad \begin{array}{lllllll}3 & 2 & 6 & 8 & 10\end{array}$

(BI) Nuestra mayor preocupación es siempre lo que es mejor para la otra persona.

$\begin{array}{lllll}4 & 0 & 0 & 8 & 5\end{array}$

(BI) En esta compañía las personas buscan el bien del otro.

$\begin{array}{lllll}0 & 6 & 15 & 8 & 0\end{array}$

(BC) En esta compañía se espera que usted hará siempre lo que es correcto para los consumidores y para el público.

$\begin{array}{lllll}0 & 0 & 0 & 24 & 20\end{array}$

(EC) El modo eficiente es siempre el modo correcto en esta compañía.

$\begin{array}{lllll}0 & 0 & 18 & 4 & 5\end{array}$

(EC) En esta compañía se espera que cada persona trabaje eficientemente por encima de todo.

$0 \quad 0 \quad 6 \quad 16$

2. Leyes y códigos profesionales (law and code)

(PC) Se espera que las personas se sujeten a las leyes y a los patrones profesionales, por encima de otras consideraciones.

$\begin{array}{lllll}0 & 0 & 6 & 4 & 15\end{array}$

(PC) En esta compañía, la ley o el código de ética de la profesión es la mayor preocupación.

$\begin{array}{lllll}0 & 0 & 3 & 16 & 10\end{array}$

(PC) En esta compañía, se espera que las personas sigan $\begin{array}{llllll}\text { estrictamente las normas legales o profesionales. } & 0 & 0 & 0 & 12 & 25\end{array}$

(PC) En esta compañía, la principal preocupación es si alguna decisión viola alguna ley.

\section{Reglas (rules)}

$(\mathrm{PL})$ Aquí es muy importante seguir las reglas y procedimientos de la compañía.

$\begin{array}{lllll}0 & 0 & 9 & 8 & 20\end{array}$

$(P L)$ Se espera que todos se mantengan fieles a las reglas y procedimientos de la compañía.

$\begin{array}{lllll}0 & 0 & 9 & 0 & 25\end{array}$

(PL) Las personas de éxito de esta compañía son aquellas que son correctas.

$\begin{array}{lllll}1 & 0 & 9 & 4 & 10\end{array}$

(PL) Las personas en esta compañía obedecen estrictamente las políticas de la compañía.

$\begin{array}{lllll}0 & 0 & 9 & 8 & 15\end{array}$




\section{Conjunto de instrumentos o medios}

(EI) En esta compañía, las personas protegen sus propios intereses por encima de cualquier cosa. $\begin{array}{llllllll}4 & 0 & 3 & 8 & 5\end{array}$

(EI) En esta compañía, las personas valen más por ellas mismas.

$2 \quad 2 \quad 3 \quad 8 \quad 5$

(EI) En esta compañía no hay espacio para principios morales, éticos y personales.

(EL) Se espera que las personas hagan cualquier cosa para promover los intereses de la compañía, sin considerar sus consecuencias.

$\begin{array}{lllll}0 & 2 & 3 & 4 & 0\end{array}$

Las personas aquí se preocupan por los intereses de la empresa, excluyendo todo lo demás.

$\begin{array}{lllll}1 & 0 & 9 & 0 & 0\end{array}$

(EL) El trabajo es considerado inferior al estándar, solamente cuando afecta los intereses de la compañía.

(EC) En esta compañía la principal responsabilidad de las personas es controlar los costos.

\section{Independencia}

(PI) En esta compañía se espera que las personas sigan sus propias creencias personales y morales. $\begin{array}{llllll}1 & 0 & 0 & 12 & 15\end{array}$

(PI) En esta compañía cada persona decide por sí misma lo que es correcto o lo que es erróneo. $\quad \begin{array}{llllll}1 & 0 & 0 & 0 & 10\end{array}$

(PI) En esta compañía la mayor preocupación es el sentido de lo correcto y errado de cada persona. $\begin{array}{llllll}0 & 6 & 12 & 9 & 0\end{array}$

(PI) En esta compañía las personas se guían por sus propias éticas personales.

$\begin{array}{lllll}1 & 0 & 9 & 4 & 10\end{array}$

*Las abreviaciones para las dimensiones teóricas del clima ético son:

$\mathrm{B}=$ Benevolencia; $\mathrm{I}$ = Individual; $\mathrm{L}=$ Local; $\mathrm{C}=$ Cosmopolita; $\mathrm{P}=$ Principio; $\mathrm{E}=$ Egoísmo.

buida en factores de 0 a 5 , como una forma de determinar los valores efectivos, es decir, cómo las cosas se encuentran en las organizaciones en este momento exacto. Se piensa que en el futuro, por la correlación entre estos dos valores, se podrá determinar cómo son las organizaciones y cómo esas deberían serlo, siguiendo el ejemplo del estudio de Kelloway et ál. (1999).
Cuadro 2: Cómo se encuentran las empresas en este momento

\begin{tabular}{lc}
\hline Ítem & Tasa media \\
\hline Interés (caring) & 3,35 \\
Leyes y códigos & 3,66 \\
Reglas & 3,91 \\
Instrumental & 1,46 \\
Independencia & 2,34 \\
\hline
\end{tabular}


Comparando este trabajo con los estudios hechos por el Departamento de Defensa Nacional del Canadá en 1998 (que utilizó una muestra de 111 miembros de su cuadro militar), se encuentra que apenas el ítem instrumental, con una media de 1,46 , quedó fuera de la media canadiense, de 3,00, como se encuentra actualmente, y de 2,33, en la forma como debería estar. En este caso, los canadienses fueron de la opinión de que la escala para el ítem instrumental tiene resultados inversos, es decir que a mayor media (factor), mayor es la percepción negativa respecto al clima ético.

Volviendo al análisis del cuadro 1, se puede observar que la mayor concentración, de los ítems se produce en los factores 3, 4 y 5, lo que puede ser interpretado, por lo menos en este momento, como diferente de los resultados obtenidos en los estudios de Victor y Cullen (1988). Vale la pena observar que el ítem interés (caring), los factores 3 y 4 tuvieron una alta calificación por parte de los encuestados, de manera que un buen número de personas centra sus intereses en la empresa y no en la cooperación con sus colegas de trabajo. Por otro lado, es razonable afirmar que por el factor 5 se puede concluir que aún hay un número importante de empleados que tienen actitudes aparentemente éticas con relación al público en general.

En cuanto al ítem leyes y códigos profesionales, quedó claro, por los resultados de la investigación, que los empleados consideran que las leyes y códigos profesionales son importantes para el desempeño de las funciones, en un sistema normativo regulado y controlado, lo que constituye la base para el buen funcionamiento de la organización y da señales de estabi- lidad personal dentro y fuera de la empresa.

Fue interesante observar la diferencia cognitiva en las opiniones de los empleados de las nueve compañías respecto a las reglas y las leyes y los códigos profesionales. Un número significativo de personas no considera que las reglas establecidas por las organizaciones son totalmente confiables; aunque aproximadamente las dos terceras partes de la muestra creen que las reglas son importantes para el buen desempeño de la empresa.

En lo concerniente al ítem instrumental, el cuadro de respuestas muestra una preocupación importante tanto por la imagen de la empresa como con relación a los intereses personales y a los de la compañía, en el aspecto de las ganancias. Un número más significativo de respuestas para este ítem señala una lectura relativamente preocupante, reflejada, por ejemplo, en la calificación dada a la expresión: «En esta compañía no hay espacio para principios morales, éticos y personales». Toda la muestra anotó como verdadera esta expresión. Sería recomendable un estudio más profundo sobre este aspecto, en las mismas empresas investigadas, para obtener un análisis más consistente.

Con relación al ítem independencia, hay dos temas relevantes para estudios posteriores, en la medida en que se manifiesta cierta falta de monitoreo o análisis por parte de las empresas. En la expresión: «En esta compañía la mayor preocupación es el sentido de lo correcto y errado de cada persona» no se obtuvo puntuación alguna. Para la expresión: «En esta compañía las personas se guían por sus propias éticas personales», llamó la atención la relativamente baja difusión de la forma 
como la ética debe ser tratada en las organizaciones, ya que un número importante de encuestados calificó la mencionada expresión con el factor número 3.

\section{Consideraciones finales}

De acuerdo con los resultados, las nueve empresas consideradas en la investigación presentan diferentes características en términos del clima ético. Fue preferible considerar la media general de cada organización a fin de reflejar de manera más amplia posible las percepciones de los empleados. Los valores incoherentes fueron dejados de lado en el cómputo general del análisis de factores. Se puede afirmar que las organizaciones tienen distintos tipos de clima ético y que también hay una cierta diferenciación en el clima ético dentro de las propias organizaciones, en función de las normas escritas o no escritas, difundidas por los directivos de cada empresa.

No fue posible realizar un estudio comparativo profundo y complejo de los resultados de las organizaciones. Cada una tiene una historia y trayectoria diferente y estas variables influyen de manera importante en los resultados generales. Se cree que se podría efectuar un análisis más profundo entre las empresas si se tomaran en cuenta la semejanza entre ellas a partir de datos como los siguientes: fecha de fundación, número de empleados, beneficios otorgados, nivel de escolaridad y rangos de edades, que no era el objetivo de este estudio.

Como ya se ha mencionado, hay diferencias de clima ético al interior de las compañías, tema que depende mucho del directorio, de la gerencia y de la supervisión del área, A pesar de esto, se levantaron datos promedios generales para cada organización, y no por departamentos o áreas. En estudios futuros se pretende establecer coeficientes de correlación entre variables dependientes e independientes, aunque se trabaje sobre una muestra menor.

Se buscó estudiar la dinámica de las relaciones interpersonales, interorganizacionales e intraorganizaciones, para comprender el clima ético existente en las empresas encuestadas, a la luz de los trabajos de investigación realizados por Victor y Cullen, los cuales influyeron en una gama de resultados organizacionales de desempeño (perfomance), satisfacción e innovación.

Los resultados alcanzados con la presente investigación no pretenden ser comparables con los estudios realizados por Victor y Cullen. Sin embargo, de alguna forma, sirven como base para futuros estudios sobre el clima ético y otros trabajos organizacionales. Se espera así que puedan contribuir significativamente al desarrollo del tema y al crecimiento sustentable de las empresas.

Ciertamente el estudio del clima ético en el trabajo se basa en el razonamiento ético individual y grupal de las organizaciones, y esto debería extenderse a la teoría de las organizaciones, a un nuevo campo de sistemas y gerenciamiento ético, a fin de transformar positivamente los escenarios. El personal de las empresas podrá resaltar sus valores y considerarlos para el enriquecimiento personal y grupal, lo que se reflejará en la imagen interna y externa de las compañías.

Entre las limitaciones del estudio, se admite que la división de la composición 
social de las empresas estudiadas podría haber sido más estructurada y reflejar un mayor detalle de los tipos humanos estudiados. Se admite también que una muestra más grande podría eventualmente cambiar los resultados obtenidos en esta investigación. Asimismo, sería conveniente un análisis matemático más sofisticado, con menos posibilidades de errores, como propone el modelo de Victor y Cullen.

El contenido del presente estudio puede ser aplicado a cualquier tipo de organización, dentro y fuera del estado de São Paulo, y puede contribuir sustancialmente para la mejora de los estudios del clima ético en el trabajo, especialmente en este momento, cuando en el Brasil la responsabilidad social y la transformación de carácter del ciudadano están adquiriendo importancia en los diversos campos de actividad, tanto de la iniciativa privada como la estatal.

Se sugiere analizar otros actores sociales, aunque de menor posición económica y social, pues en la medida en que se hagan otros estudios similares, mayor será el número de empresas que incorporarán a sus empleados en la dinámica de las relaciones compatibles con la realidad moderna, más civilizada.

Se cree que este estudio basado en las teorías de Victor y Cullen puede ser continuado, ampliado y enriquecido. Se espera que las observaciones y sugerencias sean tenidas en cuenta y abarquen otras áreas geográficas del país y un mayor número de empresas.

\section{Referencias bibliográficas}

ABDI, Hervé. 2003. Factor rotations in factor analyses. En: Lewis-Beck, M.; Bryman, A. y Futing, T. Encyclopedia of social sciences research methods. California: Thousand Oaks.

AGUILAR, Francis. 1996. A ética nas empresas. Rio de Janeiro: Zahar.

ARRUDA, Maria Cecilia Coutinho de y NAVRAN, Frank. 2000. Indicadores de clima ético nas empresas. RAE, Revista de Administração de Empresas. Vol. 40, n. ${ }^{\circ}$ 3, jul.-set. págs. 26-35.

ARRUDA, Maria Cecilia; WHITAKER, Maria do Carmo y RODRIGUEZ, Jose Maria. 2001. Fundamentos de ética empresarial e econômica. São Paulo: Atlas.
BART, Victor y CULLEN, John B. 1987. A theory and measure of ethical climate in organizations. En: Frederick, W. C. (ed.). Research in corporate performance. Greenwich, C.T.: JAI Press, págs. 57-71.

DE GEORGE, Richard. 1995. Business Ethics. $4^{\mathrm{a}}$ ed. New Jersey: Princeton Hall Inc.

FRITZCHE, David y BECKER, H. 1984. Linking management behavior to ethical philosophy. Academy of Management Journal. N. ${ }^{\circ} 27$, págs. 166-175.

JOBIM, Cynthia Maria Cirillo. 2004. Clima ético e responsabilidade social: a avaliação dos empregados sobre a relação ética das empresas com os seus stakeholders. Presentado en: Mestrado em Administração de Empresas. FGV-EAESP. 
KELLOWAY et ál. 1999. Ethical decisionmaking in DND: The development of a measurement instrument. Sponsor research report 99-14, Otawa: National Defence Headquarters. Págs. 34. [http://www. dnd.ca/ethics/dep/documents/ Sondage1_e.doc].

MERTON, Robert. 1957. Social theory and social structure. New York Press.

RAY, J. J. 1973. Factor analysis and attitude scales. The Australian and the New Zea- land. Journal of Sociology. Vol. 9, n. ${ }^{\circ} 3$, págs. 11-12.

SCHNEIDER, Benjamin. 1975. Organizational climate: An essay. Personnel Psychology. Vol. 28, págs. 447-479.

1988. The organizational bases of ethical work climates. Administrative Science Quarterly. Vol. 33, iss. 1, págs. 101-125. 